\title{
RFI STATISTICAL DISTRIBUTION AND MISSED DETECTION IN AQUARIUS RADIOMETER MEASUREMENTS
}

\author{
Paolo de Matthaeis and David M. Le Vine \\ NASA Goddard Space Flight Center \\ Greenbelt, MD, USA
}

\begin{abstract}
Aquarius is an microwave active/passive sensor whose main goal is to globally estimate sea surface salinity from space $[1,2]$. Two instruments, a radar scatterometer and a radiometer, operate at L-band observing the same surface footprint almost simultaneously. The sensitivity to sea surface salinity (SSS) is given by the radiometer, while the scatterometer measurements provide a correction for sea surface roughness. Although the primary objective is the measurement of SSS, the instrument combination operates continuously, acquiring data over land and sea ice as well. Radio Frequency Interference (RFI) can occur in both the radiometer and the scatterometer bands of operation, and for this reason detection and mitigation of RFI was included in the data processing of both active and passive instruments. This paper will focus on the RFI processing for the Aquarius radiometer only and provide an update on the efforts to reduce the amount of missed RFI detection.
\end{abstract}

Index Terms - Passive Microwave Remote Sensing, RFI, Aquarius

\section{INTRODUCTION}

The Aquarius radiometer acquires samples every $10 \mathrm{~ms}$ and averages all samples collected within a $1.44 \mathrm{~s}$ window to yield a single antenna temperature data point. The high sampling rate is much higher than required by the Nyquist-Shannon theorem allowing a number of samples affected by RFI to be discarded without significantly affecting the quality of the salinity retrieval. The detection of RFI for the Aquarius radiometer is performed in the time domain with samples that differs too much from the local mean being flagged as contaminated by RFI. RFI mitigation is achieved by removing the RFI-flagged samples from the processing chain before averaging them into a single data point. A more detailed description of the algorithms is given in [3].

The RFI algorithm successfully detects RFI in most cases, as reported in $[4,5]$. However, the RFI flags only indicate suspected RFI, which may also include false RFI detection, or "false alarms". If the same thresholds are used on ocean and land in the RFI algorithm, the level of false alarms is higher in measurements over land, where the samples have a higher standard deviation and are therefore more likely to exceed the RFI flagging threshold. For this reason, the RFI thresholds have been adjusted and now produce comparable false alarm rates over ocean and land, while sea ice and transition zones between ocean and land still have a higher level of false alarms. The main effect of a high false alarm rate is to degrade the quality of the $1.44 \mathrm{~s}$ data points, since it leads to discarding useful samples in the averaging.

A more serious problem is caused by low-level interference that can remain undetected by the RFI filter. One example occurs when strong RFI enters the antenna sidelobes and produces the same effect as low or moderate RFI coming from the main beam. If this RFI is not detected, it can cause the estimated SSS for ascending and descending track to differ. A methodology has been developed to estimate missed detection and results will be shown for some particular cases.

\section{ESTIMATION OF MISSED DETECTION}

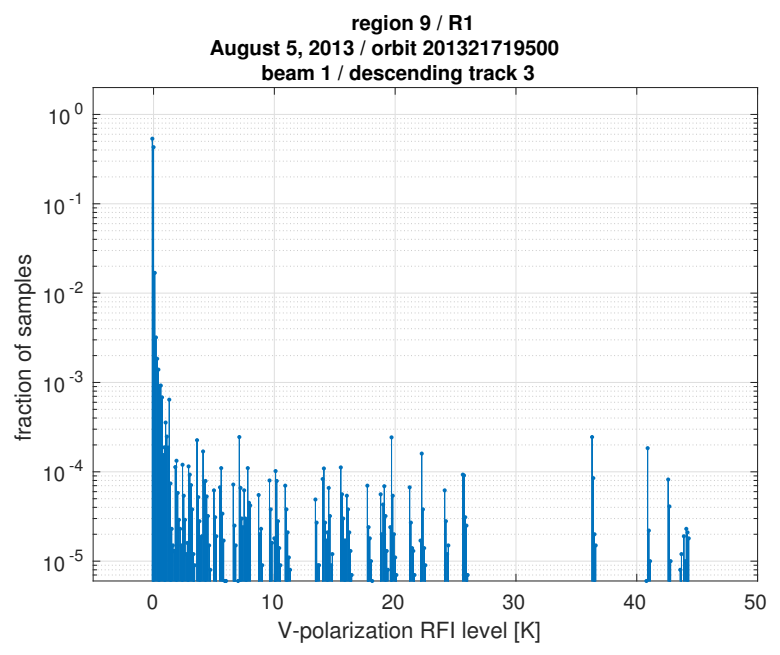

Fig. 1. Estimated histogram of RFI.

The estimation of missed detection requires the knowledge of RFI statistics. A procedure has been developed to estimate the histograms of the RFI by statistically comparing 
measurements acquired in presence of RFI and in an RFI-free environment. Figure 1 shows one of the estimated RFI histograms.

Once the RFI histograms are estimated, simulated RFI levels are generated using them, and simulated $10 \mathrm{~ms}$ antenna temperature samples without and with simulated RFI are run through the RFI filter to estimate missed detection, as illustrated in the flow diagram of Figure 2.

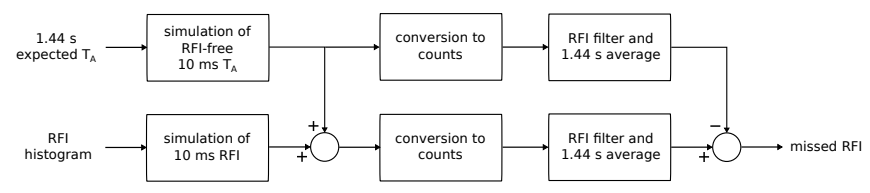

Fig. 2. Estimation of missed detection.

Details on the estimation of the RFI histograms and all the steps will be provided, with examples and discussion. For the missed detection estimate, the areaas in the North Atlantic that experienced a change in the RFI environment in 2013 due to adjustem of operating frequency of radars in Canada will be considered. Results of this missed detection estimate shows that a considerable amount of RFI below $0.1 \mathrm{~K}$ can be missed by the Aquarius.

\section{REFERENCES}

[1] D.M. Le Vine, G.S.E. Lagerloef, F.R. Colomb, S.H. Yueh, and F.A. Pellerano, "Aquarius: An Instrument to Monitor Sea Surface Salinity From Space", IEEE Transactions on Geoscience and Remote Sensing, vol. 45, no. 7, pp. 2040-2050, July 2007

[2] G.S.E. Lagerloef, F.R. Colomb, D.M. Le Vine, F. Wentz, S.H. Yueh, C.S. Ruf, J. Lilly, J. Gunn, Y. Chao, A. deCharon, G. Feldman, and C. Swift, The Aquarius/SACD Mission: Designed to meet the salinity remote-sensing challenge, Oceanography, vol. 21 no. 1, pp. 6881, March 2008.

[3] S. Misra and C. Ruf, Detection of Radio Frequency Interference for the Aquarius Radiometer, IEEE Transactions on Geoscience and Remote Sensing, vol. 46, no. 10, pp.3123-3128, October 2008.

[4] D.M. Le Vine, P. de Matthaeis, C.S. Ruf, and D.D. Chen, Aquarius RFI Detection and Mitigation Algorithm: Assessment and Examples, IEEE Transactions on Geoscience and Remote Sensing, vol.52, no.8, pp.4574-4584, Aug. 2014.

[5] D.M. Le Vine and P. de Matthaeis, Aquarius Active/Passive RFI Environment at L-Band, IEEE Geoscience and Remote Sensing Letters, vol.11, no.10, pp.1747-1751, Oct. 2014. 